\title{
Real Time Micro-Fiberoptic Monitoring of Endogenous Fluorescence in the Rat Conceptus During Hypoxia
}

\author{
BJORN A. THORSRUD AND CRAIG HARRIS \\ Toxicology Program, Department of Environmental and Industrial Health, \\ University of Michigan, Ann Arbor, Michigan 48109
}

\begin{abstract}
A micro-fiberoptic methodology has been developed for noninvasive, real time measurement of endogenous pyridine nucleotide fluorescence from the surface of the visceral yolk sac (VYS) in intact, viable rat conceptuses. Gestational day (GD) 10-12 conceptuses are maintained in a customized perifusion system, which allows for control of oxygenation, as well as the continuous measurement of $\mathrm{pH}$ and oxygen concentration in the effluent perifusate. Miniaturized light guides were constructed by drawing $250 \mu \mathrm{m}$ ESKA acrylic optical fibers through a stainless steel sheath with a high strength epoxy polymer. A single fiber supplied the excitation signal from a mercury arc lamp at a wavelength of $366 \mathrm{~nm}$. The emission signal was returned via three additional fibers, electronically amplified, processed, and recorded, using a dual channel lamp-compensated fluorometer, optimized for detection of reduced pyridine nucleotides at $455 \mathrm{~nm}$. Endogenous fluorescence in the conceptus was monitored by placing the polished tip of the sensor directly on the surface of the VYS. Oxygen-equilibrated conceptuses, exposed to $100 \%$ nitrogen, produced a reproducible biphasic surface fluorescence peak, which returned to baseline levels upon reoxygenation of the perifusate. This biphasic response consisted of an initial rapid rise in fluorescence (phase $\mathbf{I}$ ), followed by an attenuated rate in fluorescence signal increase (phase II). The hypoxia produced age-dependent rates of fluorescence change during phase I, while phase II remained relatively unchanged throughout GD 10-12. These results demonstrate the ability to monitor endogenous fluorescence, non-invasively and in real time, during the period of organogenesis in the intact rat conceptus and will provide valuable information in studies of embryonic metabolism and response to chemical embryotoxicants. 1993 Wiley-Liss, Inc.
\end{abstract}

The ability to elucidate mechanisms of teratogenesis has been hampered by the need to superimpose the biochemical and physiological disturbances elicited by chemicals and environmental factors, over an already complex and poorly understood developmental program. It is becoming clear that many mechanisms of teratogenesis may also involve alterations of several interrelated pathways including those responsible for metabolism, energy production, and cellular protection. Recent reports have focused on the role of oxygen concentration and the cellular selectivity for chemical toxicity based on the ability to deal with abnormally low or high oxygen concentrations during different phases of embryogenesis
(Fantel et al., '89, '91). While the direct effects of altered oxygen concentration have been shown to play an important role in determining embryotoxicity, other consequences related to alterations in metabolism and energy production may also produce equally detrimental effects.

One particular area of concern during development is the flux of cellular reducing equivalents, in the form of the pyridine nucleotides $[\mathrm{NAD}(\mathrm{P}) \mathrm{H}]$, which are vital for

\footnotetext{
Received January 13, 1993; accepted June 14, 1993.

Address reprint requests to Dr. Craig Harris, Toxicology Program, Department of Environmental and Industrial Health 1420 Washington Heights, University of Michigan, Ann Arbor MI 48109-2029.
} 
biosynthesis, energy production, and maintenance of cellular homeostasis. Demonstrations of high metabolic and detoxicative capacity in the rodent visceral yolk sac (VYS), including those involving cytochrome $\mathrm{P}_{450}$ monooxygenases, also involve the flux of pyridine nucleotides and suggest that the dynamic relationship between the embryo and its extraembryonic membranes may be of critical importance in determining the overall consequences of exposure to chemicals or extreme environmental changes. A micro-fiberoptic monitoring procedure capable of detecting alterations in pyridine nucleotide status in intact, viable conceptuses (conceptus, in this report refers to the intact embryo, its associated extraembryonic membranes, and the ectoplacental cone, as prepared for whole embryo culture), noninvasively and in real time, greatly enhances the ability to evaluate the importance of such alterations during normal embryogenesis and, as a consequence, of xenobiotic insult.

Using a two-fiber micro-light guide, Ji et al. (79) showed that it was possible to measure changes in NADH fluorescence from the surface of an intact liver. Over the subsequent years this technology has been further refined, particularly in the laboratory of R. G. Thurman ( $\mathrm{Ji}$ et al., '80; Thurman and Lemasters, '88), and applied to studies concerning the dynamic functional differences that exist between periportal and pericentral hepatocytes, including differences in intermediary metabolism, bioactivation, detoxication, lipid metabolism, glutathione (GSH) conjugation, and others (Thurman and Lemasters, '88; Belinsky et al., '85; Olson and Thurman, ' 84 ; Harris and Thurman, '86).

The basic principle of micro-fiberoptic operation (using endogenous pyridine nucleotide fluorescence as an example) involves placement of two or more optical fibers against the surface of the tissue to be evaluated. Illumination of the surface by fibers emanating from a filtered $366 \mathrm{~nm}$ mercury arc lamp source excite NADH and NADPH, eliciting a broad visible fluorescence peak at $455 \mathrm{~nm}$. This signal is transmitted via one or more collection fibers to a photomultiplier for detection and subsequent electronic amplification and recording. In normally aerobic tissues, such as the liver, $\mathrm{NAD}(\mathrm{H})$ is found predominantly in the oxidized form while NADP $(H)$ remains primar- ily in the reduced form (Sies, '82). Only the reduced pyridine nucleotides will fluoresce when excited at $366 \mathrm{~nm}$. NADH is oxidized primarily in the respiratory chain of the mitochondria by a mechanism dependent on the concentration of molecular oxygen. When oxygen is removed from the system $\mathrm{NAD}^{+}$becomes reduced (to $\mathrm{NADH}$ ), but not until the tissues become almost entirely anoxic (Chance et al., '62). Since NADPH is already in the reduced state, it is believed that virtually all increases in endogenous pyridine nucleotide fluorescence detected by the light guides are due to the increase in NADH. Intracellular levels of both $\operatorname{NADP}(\mathrm{H})$ and $\operatorname{NAD}(\mathrm{H})$ are high in many cell types [about $0.5 \mathrm{mM}$ in the liver (Thurman and Lemasters, '88)] and conditions affecting either the increase or decrease in total reduced nucleotide should be detectable by the surface fluorescence probe. Very little is known about the flux of pyridine nucleotides in the conceptus during embryogenesis, including possible spatial and temporal differences that may contribute to cell- or tissue-selective embryotoxicity.

The ability to monitor pyridine nucleotide changes non-invasively in the intact developing conceptus will provide a number of powerful advantages for the study of developmental processes during organogenesis and the effects of chemicals and environment on those processes. The changes associated with the transition from anaerobic to aerobic metabolism (Freinkel et al., '84) during critical early periods of neurulation, heart, craniofacial, and limb development, make superimposed chemically induced effects which alter metabolism and cellular oxidation state very difficult to interpret. This is especially true when only static measurements can be made using disrupted tissue samples. The ability to monitor one or more relevant processes such as pyridine nucleotide status in real time and in an intact conceptus will help in understanding the effects of toxic chemicals and oxidative injury in terms of the dynamic changes that may be occurring over time. This approach will prove to be very useful in the evaluation of chemically-induced changes in pyridine nucleotide status during anoxia and hyperoxia, during disruption of intermediary metabolism and mitochondrial function, during poly-(ADP-ribose) polymerase activity, and as a consequence of the metabolism of model xenobiotics. 


\section{MATERIALS AND METHODS Animals}

Time mated, primigravida Sprague Dawley rats are obtained from the University of Michigan Reproductive Sciences Program, P-30 Small Animal Core facility, where the animals are maintained in a $10 \mathrm{~h} / 14 \mathrm{~h}$ light dark cycle, the dark cycle beginning at 5 pm. Food and water are provided ad libitum. One male is caged with two females in the late afternoon, and the following morning a vaginal smear is used to confirm pregnancy. This is designated as day 0 of gestation.

\section{Whole rat embryo culture}

On gestation day (GD) 10, pregnant dams are ether anesthetized and the rat conceptuses explanted using routine explantation protocols, as described previously (Hiranruengchok and Harris, '93). The explanted embryos are then grown in culture media composed of $33 \%$ heat inactivated female rat serum in Hank's Balanced Salt Solution (HBSS), at pH 7.4 (Gibco, Grand Island, NY). Growth parameters are described in detail in Freeman et al. ('87).

\section{Perifusion system}

A customized perifusion system was designed to maintain the viability of GD $10-12$ rat conceptuses in serum-free medium for up to $2 \mathrm{hr}$ of fiberoptic monitoring. It was important to construct a system that could maintain constant flow, temperature, $\mathrm{pH}$, dissolved oxygen concentration, control chemical exposure, permit observation of probe placement, and constantly monitor embryo viability (heart rate and VYS vascular flow). The entire perifusion system is shown schematically in Figure 1. Earle's Balanced Salt Solution (EBSS) was used as perifusate. The EBSS working solution is made up fresh each day, by diluting a stock $10 \times$ solution (Earle et al., ' 43 ) to a $1 \times$ concentration and adjusting the $\mathrm{pH}$ with sodium bicarbonate to pH 7.55. EBSS is delivered at a flow rate of $2 \mathrm{ml} / \mathrm{min}$ from a $250 \mathrm{ml}$ media reservoir via a TRIS peristaltic pump (ISCO) to an artificial lung. The customized artificial lung is constructed using a sealed glass jar, with a filtered gas inlet for oxygenation or deoxygenation and a small purge outlet. The perifusate is pumped through coiled, gas permeable medical grade silicone tubing made of Silastic Q74750 (Baxter, McGaw Park, IL), I.D. 0.058" $\times$ O.D. $0.077^{\prime \prime}$, which insures rapid equilibration of culture medium with ambient nitrogen and the various oxygen concentrations (Berry et al., '74). Gassed media is warmed to $37^{\circ} \mathrm{C}$ as it passes through an insulated, heated water jacket prior to entering the fiberoptic monitoring chamber. The effluent perifusate is then directed to flowthrough oxygen and $\mathrm{pH}$ monitors (Lazar, CA), which allow for continuous monitoring of both oxygen concentration and $\mathrm{pH}$. These are calibrated to the media, prior to the addition of the rat conceptus, and signals are continuously and simultaneously recorded throughout the experiment. The monitoring chamber itself is constructed from clear, acrylic polymer, with a $4 \mathrm{~mm}$ diameter and $5 \mathrm{~mm}$ deep well, supplied by warmed media from an inlet in the bottom and removed from an outlet near the top (see Fig. 2). Small black glass beads $(0.5 \mathrm{~mm}$ diameter) fill the bottom of the chamber and provide a bed to support the conceptus and through which the perifusate can evenly diffuse during fiberoptic monitoring. The chamber is fitted with a resealable rubber septum at the top that allows for easy insertion and removal of the stainless steel-sheathed fiberoptic probe. Additionally, it allows for stability, precise positioning of the microlight guide during monitoring, and also provides a means to easily remove air bubbles trapped in the chamber. Recent modifications permit placement of the monitoring chamber on the rotating stage of a reclined slide microscope with a $3.5 \times$ objective lens. This enables more precise placement of the fiberoptic probe and simultaneously allows for constant monitoring of heart rate and visualization of the VYS vascular flow during the experimental period.

\section{Fiberoptic system}

The fiberoptic system is optimized for detection of reduced pyridine nucleotides (NADH, NADPH) from the surface of the rat VYS, at an excitation wavelength of 366 $\mathrm{nm}$ and emission wavelength of $455 \mathrm{~nm}$. The micro-fiberoptic probes are constructed by drawing seven $250 \mu \mathrm{m}$ ESKA acrylic fibers (Edmund Scientific Co., Barrington, NJ) through an 18 gauge stainless steel hypodermic needle, using a high strength polymer epoxy (West System Epoxy, Bay City, MI). The tip is polished to a microscopically even finish with jeweler's rouge. These materials proved to be relatively easy 


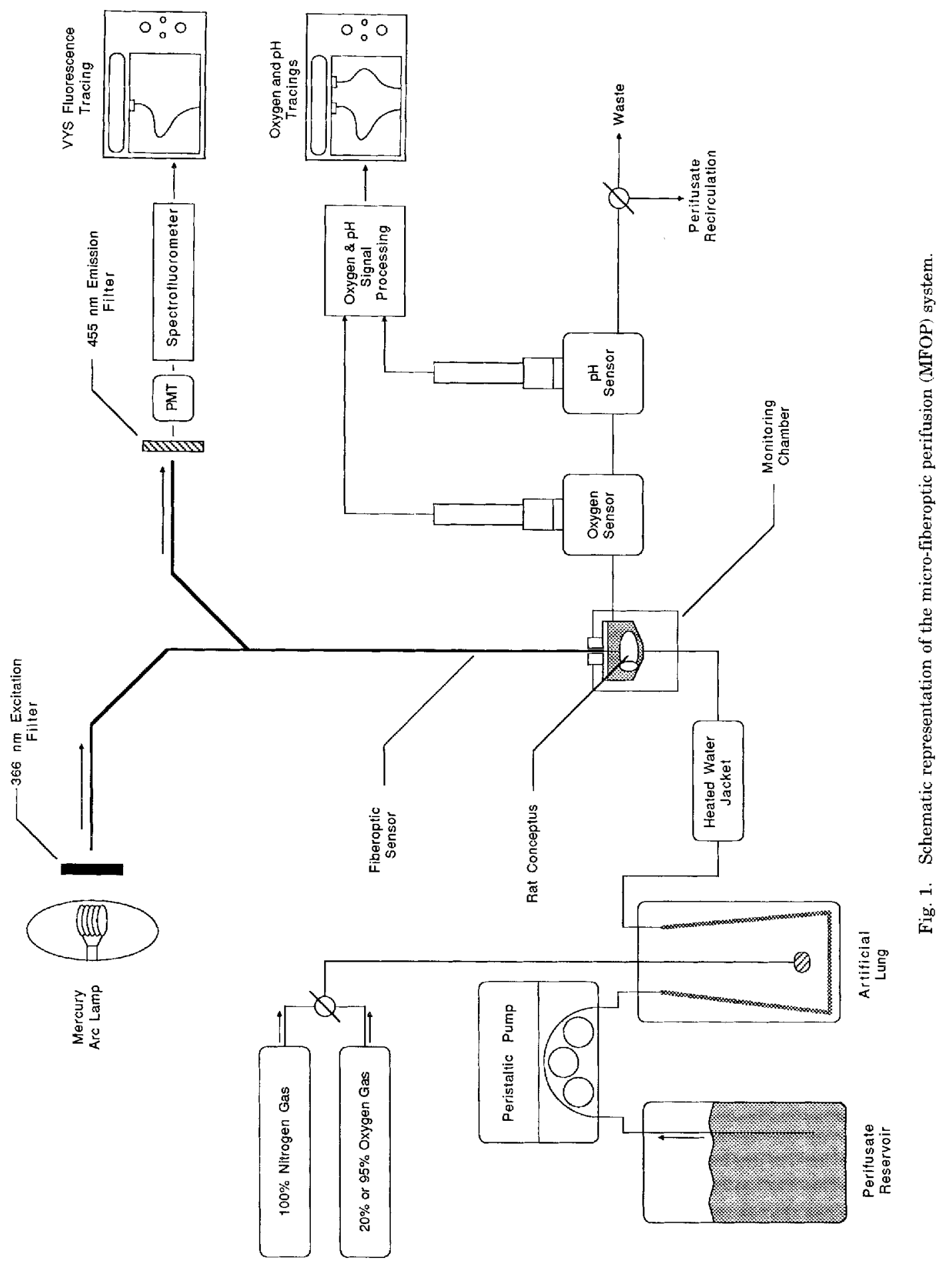




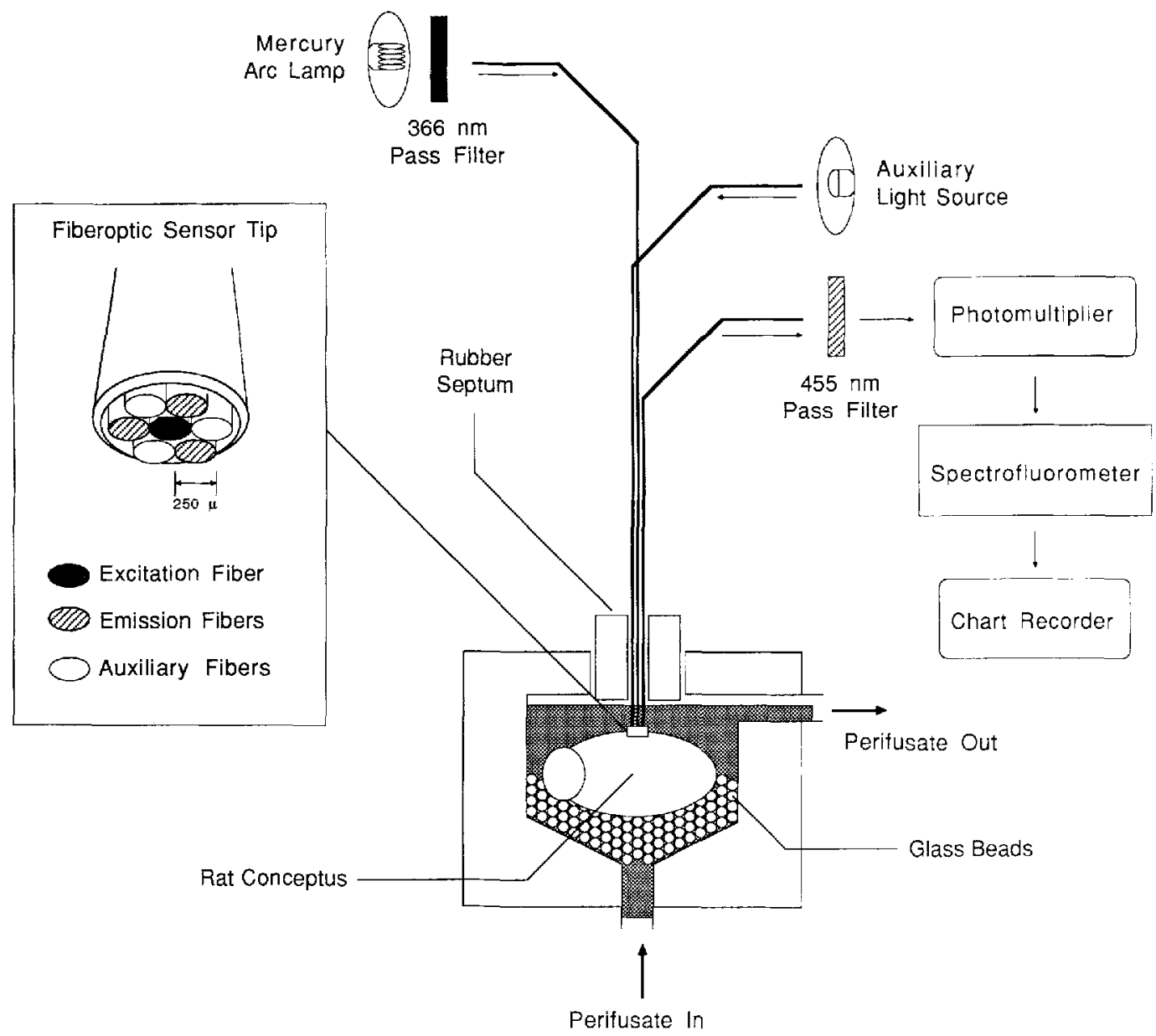

Fig. 2. Schematic diagram of the MFOP monitoring chamber, showing the location of the rat conceptus in the chamber and the placement of the sensor on the surface of the VYS. Insert shows amplified view of the micro-fiberoptic sensor tip.

to manipulate during probe fabrication and result in excellent fluorescence conductance. The fiber configuration is relatively simple (insert, Fig. 2); a 100 W Nikon mercury arc lamp with a $366 \mathrm{~nm}$ cutoff filter (Corning glass filter no. 5840) provides the excitation signal directed through a single fiber located in the middle of the bundle to the surface of the VYS. Three alternating fibers surrounding the excitation strand, gather the surface fluorescence signal from the VYS and guide it through a $455 \mathrm{~nm}$ bandpass filter (Kodak Wratten gelatin filters $2 \mathrm{E}, 47$ ), to a photomultiplier (PMT) for detection. It is then amplified and processed by a dual channel lamp compensated fluo- rometer, model DCF-O, (Biomedical Instrumentation Group, University of Pennsylvania). The resultant signals are finally recorded on a strip chart recorder (Linear) at a chart speed of $20 \mathrm{~cm} / \mathrm{hr}$. The three remaining fibers can be illuminated with a visible incandescent light source during setup to help with probe placement. Routinely, the polished end of the 7-strand guide is gently positioned on the surface of the VYS of a GD 10-12 conceptus resting on its side in the micro-fiberoptic perifusion (MFOP) chamber. The total volume of the VYS actually being monitored this way is equivalent to the intersection of the cone of light emitted from the one excitation fiber 
TABLE 1. Gestational day 11.5 assessment of morphologic parameters and malformations of control rat conceptuses cultured in Earle's Balance Salt Solution for 2 hours on gestational day 10

\begin{tabular}{|c|c|c|c|c|c|c|c|c|c|}
\hline \multirow[b]{2}{*}{ Treatment } & \multirow{2}{*}{$\begin{array}{c}\% \\
\text { (viablef } \\
\text { total) }\end{array}$} & \multirow{2}{*}{$\begin{array}{l}\text { Crown- } \\
\text { Rump } \\
\text { (mm)* }\end{array}$} & \multirow[b]{2}{*}{ Somites* } & \multicolumn{2}{|c|}{ \% abnormal } & \multicolumn{2}{|c|}{ Protein content* } & \multicolumn{2}{|c|}{ DNA content* } \\
\hline & & & & $\begin{array}{c}\text { Open } \\
\text { neural tube }\end{array}$ & $\begin{array}{c}\text { Rotation } \\
\text { defects }\end{array}$ & $\begin{array}{c}(\mu \mathrm{g} / \\
\text { embryo) }\end{array}$ & $\begin{array}{c}(\mu g i \\
\text { VYS }) \\
\end{array}$ & $\begin{array}{c}\left(\mu g^{\prime}\right. \\
\text { embryo) }\end{array}$ & $\begin{array}{c}(\mu \mathrm{g}) \\
\text { VYS }) \\
\end{array}$ \\
\hline Control & $\begin{array}{c}100.0 \\
(5 / 5)^{* * *}\end{array}$ & $3.6 \pm 0.04$ & $22.8 \pm 0.4$ & 0.0 & 0.0 & $278.9 \pm 10.8$ & $183.4 \pm 7.1$ & $20.3 \pm 1.0$ & $6.1 \pm 0.1$ \\
\hline $\begin{array}{l}\text { EBSS } \\
\text { Exposed }\end{array}$ & $\begin{array}{l}100.0 \\
(9 / 9)\end{array}$ & $3.6 \pm 0.04$ & $23.0 \pm 0.3$ & 0.0 & 0.0 & $294.5 \pm 7.1$ & $202.6 \pm 6.5$ & $20.2 \pm 0.9$ & $5.7 \pm 0.3$ \\
\hline
\end{tabular}

*Values represent mean \pm SE. No significant difference was found between control and EBSS treated values $(P<0.05)$.

**Number of viable conceptuses per total number examined.

to the theoretical cones that are visualized by the three emission fibers. Using the following reference sources (Kapany, '67; Ji et al., '79; Gillet, '81;), we have calculated the total volume to be approximately $200 \mathrm{pL}$.

\section{EBSS exposure (morphology and viability assessment)}

GD 10 rat conceptuses were exposed to serum-free perifusate (EBSS, $20 \% \mathrm{O}_{2}, 5 \%$ $\mathrm{CO}_{2}, \mathrm{pH} 7.4,37^{\circ} \mathrm{C}$ ) for $2 \mathrm{hr}$, returned to standard culture medium, and assessed the next day for viability, changes in growth parameters, and presence of malformations (Hiranruengchok and Harris, '93). Conceptuses were divided into two groups and both were equilibrated for $1 \mathrm{hr}$ in standard culture media (33\% rat serum in HBSS). The control group continued incubation in fresh culture media for $2 \mathrm{hr}$, while the test group was grown for the same time under identical conditions using serum-free EBSS. Both groups were then returned to standard culture media and grown overnight as described above. A morphologic assessment for viability, growth parameters, and presence of malformations was conducted on the following day (GD 11.5) and the results are shown in Table 1.

\section{Nitrogen exposure}

Prior to fluorescence monitoring, the conceptuses are maintained in rat whole embryo culture as previously described. At the appropriate time the conceptus is carefully removed from culture and placed in the MFOP chamber. Conceptuses freshly explanted on GD 10 are allowed to equilibrate for at least $1 \mathrm{hr}$ in the culture media before they are removed and placed into the monitoring chamber. Oxygenated EBSS is allowed to circulate for a 10 min equilibration period, after which the oxygen is turned off and nitrogen is allowed to saturate the perifusate for an additional $10 \mathrm{~min}$. The surface fluorescence, $\mathrm{pH}$, and oxygen tension are simultaneously monitored during the entire period. At the end of the nitrogen exposure, the conceptus continues to be monitored while it is allowed to re-equilibrate with oxygen for an additional $10 \mathrm{~min}$.

\section{RESULTS}

The MFOP system was developed in two stages. The perifusion apparatus was constructed first and included the development, testing, and verification of physiologic parameters needed to maintain the viability of the whole rat conceptus during an extended period of observation.

Initially, we used standard embryo culture media as perifusate $(33 \%$ rat serum in HBSS), but it was found to be difficult to work with due to its relatively high viscosity and propensity to develop air bubbles in the tubing. A number of serum-free preparations were tested as perifusate. HBSS, used under normoxic conditions $\left(20 \% \mathrm{O}_{2}\right.$, $5 \% \mathrm{CO}_{2}, 75 \% \mathrm{~N}_{2}$ for day 10 and $95 \% \mathrm{O}_{2}, 5 \%$ $\mathrm{CO}_{2}$ for day 11-12), rapidly became acidic upon oxygenation ( $\mathrm{pH}$ 6.6-6.8), making it unsatisfactory as perifusate. EBSS, on the other hand, is known to possess improved acid buffering capacity over HBSS (Todd et al., '79), and resulted in the maintenance of $\mathrm{pH}$ in a physiologic range ( $\mathrm{pH} 7.4-7.5)$ during the normoxic monitoring period. Conceptuses monitored under these conditions for up to $2 \mathrm{hr}$ and then returned to normal culture conditions retained viability and showed no apparent ill effects (see Table 1).

Maintaining a physiological temperature of $37^{\circ} \mathrm{C}$ in the monitoring chamber is important for normal conceptal viability and function. Attempts to prewarm the media in a heated water bath proved unsatisfactory, because of excessive temperatures necessary to compensate for heat loss in transit. An insulated, heated water jacket was developed and placed just prior to the chamber 
inflow valve, which resulted in the ability to maintain the conceptus at a constant temperature of $37^{\circ} \mathrm{C}$.

In order to insure proper positioning of the rat conceptus in the MFOP chamber, prototype designs employed the use of a spiraled, tungsten wire basket, suspended in the chamber, with an inlet on the bottom right side of the well and an outlet on the top of the same side. This proved to be unsatisfactory, because the flow of the perifusate formed a hydrodynamic vortex around the conceptus and did not allow for proper mixing of chemicals or adequate surface exposure of the VYS. As a result, a chamber was developed with an inlet at the bottom, covered with small, black glass beads for more uniform diffusability of the perifusate, and an outlet placed at the top of the well (see Fig. 2). This eliminated the diffusion problem and allowed for improved tissue exposure.

On completion of the perifusion system, the second stage of development was initiated. This entailed the construction and testing of the micro-fiberoptic sensors. Various combinations and sizes of glass and acrylic fibers were tested. Originally, bundles of $50 \mu \mathrm{m}$ diameter glass fibers numbering 2-140 were drawn through 18-21 gauge stainless steel sheaths with 5 minute epoxy and the ends polished to an even finish with high grain emery paper and jeweler's rouge. Fluorescence intensity using these materials and configurations was poor and microscopic evaluation of the tip revealed an irregular and pitted surface that we were unable to improve. These micro-light guides were constructed in a manner similar to probes used for monitoring sublobular liver function (Ji et al., '80; Harris and Thurman, '86). Subsequent experimentation determined the fibers of choice to be $250 \mu \mathrm{m}$ diameter ESKA acrylic fibers, used in bundles of seven, which resulted in improved ease of fabrication and excellent fluorescence signal conductance.

Following final verification of the fiberoptics, the two systems were integrated and preliminary experimentation initiated using live, intact rat conceptuses. A single conceptus was carefully placed in the MFOP chamber, lying on its side on a bed of glass beads. The top was sealed with a rubber septum and the polished end of the fibersensor was gently placed on the surface of the VYS.

Initial fluorescence readings from the surface of the VYS showed a steady baseline fluorescence during a 40 min monitoring period, while bathed in oxygenated, serumfree EBSS (see Fig. 3A). Arbitrary fluorescence units (AFUs) were measured and standardized to a $1 \mathrm{~mm}$ per $30 \mu$ volt deflection on the chart paper. The presence of heart beat and VYS vascular flow were monitored before and after exposures, as an indicator of viability. A new conceptus was routinely equilibrated for $10 \mathrm{~min}$ in normoxic perifusate, which was then saturated with $100 \%$ nitrogen, and within $1 \mathrm{~min}$ of exposure a reproducible biphasic fluorescence peak was recorded. This biphasic response consisted of an initial rapid rise in fluorescence (phase I), lasting about $2 \mathrm{~min}$, followed by an attenuated fluorescence increase, termed phase II. When the perifusate was reoxygenated, the endogenous fluorescence returned to pre-exposure levels, indicating a reversible process. This phenomena, as well as $\mathrm{pH}$ and oxygen levels, were continuously monitored in GD 10-12 rat conceptuses. Representative profiles are shown in Figure 3B. The following values represent the calculated rates of AFU change as mean $1 \mathrm{SE}$ (the appropriate n-numbers are shown on the $y$-axis of Fig. 4). For phase I, the mean rates of surface fluorescence increase in GD 10,11, and 12 conceptuses were $25.8 \pm 1.4,18.7 \pm 3.6$, and $12.5 \pm 5.1 \mathrm{AFU} / \mathrm{min}$, respectively. A considerably smaller change was detected during phase II, ranging from $4.5 \pm 0.6,3.7 \pm 0.1$, and $3.9 \pm 0.4 \mathrm{AFU} /$ minute for GD 10-12 rat conceptuses, respectively. For comparisons of the rate of fluorescence increase between phase I and phase II in conceptuses of ascending age see Figure 4. The ratios of phase I to phase II were also calculated and showed a steady decrease from $5.7,5.1$, to 3.2 in GD 10,11 , to 12 conceptuses, respectively. This decreasing trend is primarily due to the initial rapid rise of endogenous fluorescence emanating from the surface of the VYS. The maximum fluorescence response after $10 \mathrm{~min}$ of nitrogen exposure resulted in a consistent and reproducible fluorescence amplitude deflection for each age group. The calculated means $\pm \mathrm{SE}$ for the maximum fluorescence signal deflection in GD 10-12 conceptuses were $4.0 \pm 0.2,2.6 \pm$ 0.2 , and $2.7 \pm 0.2$, respectively (amplitude data were obtained from conceptuses shown in Fig. 4). Conceptual age confers distinct response characteristics to the nitrogen in- 

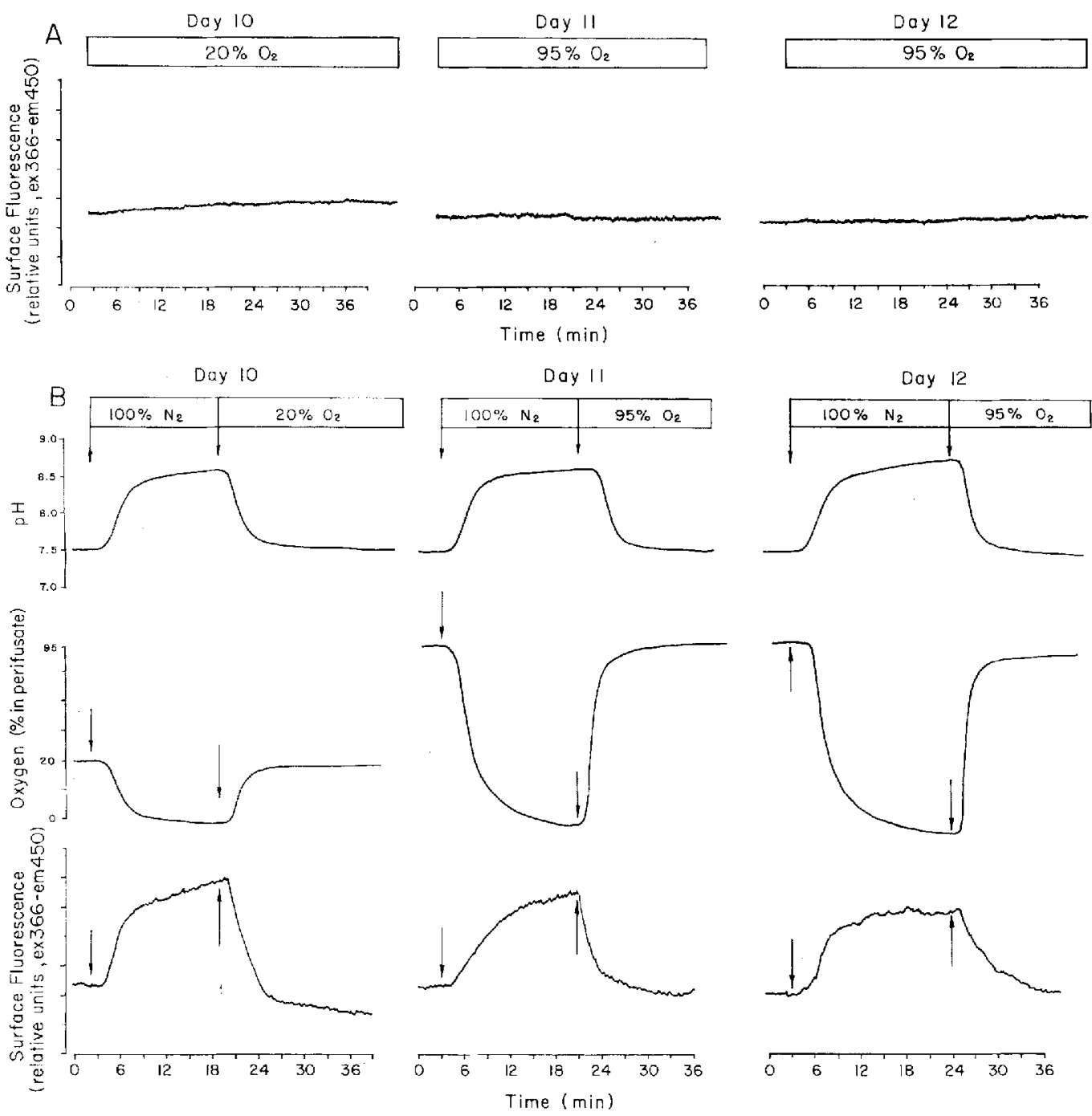

Fig. 3. A: Recordings representing baseline fluorescence levels from the surface of the VYS in GD 10-12 rat conceptuses during normoxic conditions $\left(20 \% \mathrm{O}_{2}\right.$ for GD $10,95 \% \mathrm{O}_{2}$ for GD 11-12). B: Simultaneous real time recordings of oxygen concentration and $\mathrm{pH}$ in the effluent perifusate, and fluorescence from the surface of the rat VYS in GD 10-12 rat conceptuses. The oxygen monitor was calibrated by infusion of perifusate equilibrated with $20 \% \mathrm{O}_{2}, 95 \% \mathrm{O}_{2}$, and $100 \% \mathrm{~N}_{2}$ prior to introduction of the conceptus. 'The $\mathrm{pH}$ monitor was, like-

wise, calibrated in $\mathrm{pH}$ standard solutions of $\mathrm{pH} 7.0$ and $\mathrm{pH}$ 10.0. Surface fluorescence was determined as described in Methods, where full-scale deflection, sensitivity, blank suppression, band width, and amplification settings were held constant to allow for interconceptal comparisons. The initial rapid rise in fluorescence of the representative biphasic peak during the nitrogen exposure is termed phase I, while the secondary slower rise in fluorescence is termed phase II.

duced hypoxia. This is very important, since the rat embryo undergoes a transition from anaerobic to aerobic energy metabolism during this period of organogenesis from GD 10-12 (Freinkel et al., '84).

The emission signal was standardized by

always laying the rat conceptus on its side, gently placing the probe on the surface and avoiding the polar ends, which consisted of the maternally derived ectoplacental cone on one end and the protrusion of the great vessels on the other. Heart rate and VYS 


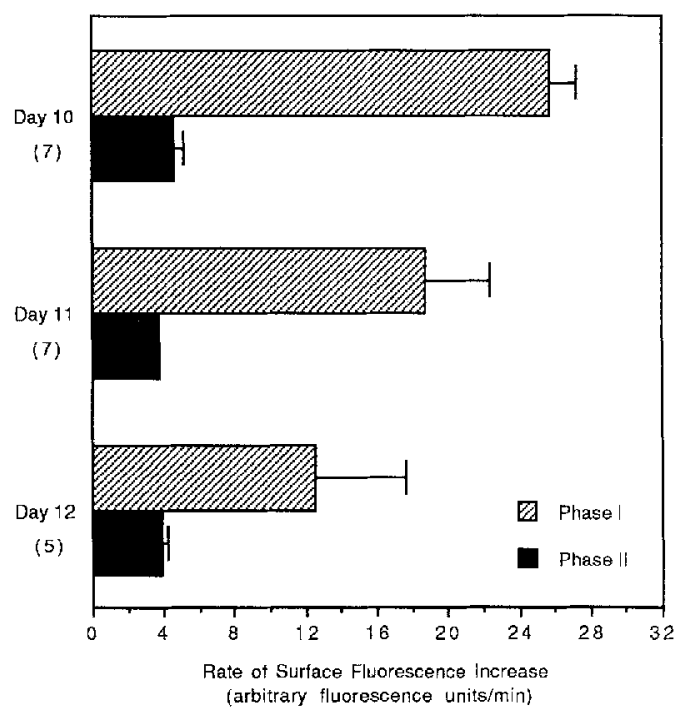

Fig. 4. Comparison of the mean rate of rise of the fluorescence signal between phase I and phase II, in GD $10-12$ rat conceptuses, during exposure to nitrogen induced hypoxia. Phase I was calculated by measuring the mean rate of rise $( \pm \mathrm{SE})$ of the fluorescence signal over time and determined to be $25.8 \pm 1.4,18.7 \pm 3.6$, and $12.5 \pm 5.1 \mathrm{AFU} / \mathrm{min}$ for GD 10,11 , and 12 rat conceptuses, respectively. Phase II was measured in the same way and was found to be $4.5 \pm 0.6,3.7 \pm 0.1$, and $3.9 \pm$ $0.4 \mathrm{AFU} / \mathrm{min}$ for GD $10-12$ conceptuses, respectively. $\mathrm{N}$-numbers are represented within parentheses.

vascular flow rate were also important indicators of fluorometric response during the nitrogen exposure. Embryos with poor circulatory parameters (heart rate and VYS vascular flow), generally responded with much lower amplitude, or did not respond at all, to the nitrogen induced hypoxia.

Many studies using fiberoptic technology have focused attention on such tissue types as rat liver (Thurman and Lemasters, '88; Harris and Thurman, '86), human muscle (Guezennec et al., '91), rabbit hearts (Foster et al., '84), and rat hearts (Minezaki et al., '90), and have confirmed increases in NADH fluorescence with hypoxia, but this is the first time that this dynamic hypoxic response has been evaluated during the organogenic period in the intact rat conceptus.

\section{DISCUSSION}

The MFOP system has shown the ability to maintain viability of an intact rat conceptus for up to $2 \mathrm{hr}$ of fiberoptic monitoring in serum-free media. It was possible to measure endogenous fluorescence in real time, non-invasively, from the surface of the VYS in the rat conceptus, during the period of organogenesis, while exposed to normoxic and hypoxic environmental conditions. Organogenesis stage conceptuses of different gestational age (GD 10-12) were monitored with a single fiberoptic sensor, using identical equilibration and exposure protocols. A reproducible biphasic fluorescence peak was recorded in GD 10-12 rat conceptuses and the initial rate of increase in tissue surface fluorescence (phase I) was found to decrease with ascending age (Fig. 4). The amplitude of response also appears to decrease with ascending age and may be related to reported decreases in VYS endoderm volume density (Gupta et al., '82). As previously mentioned, this increase in endogenous fluorescence is believed to be due to the reduction of $\mathrm{NAD}^{+}$in aerobically active tissues (Chance et al., '62). The pattern of fluorescence increase was found to be similar on all 3 gestational days monitored and appears very similar to results obtained from the surface of adult rat skin (Pappajohn et al., '72) and the isolated perfused adult rat liver ( $\mathrm{Ji}$ et al., ' 80 ; Thurman and Lemasters, '88). Although there are differences in the rate of fluorescence rise between GD 10-12 conceptuses, the overall similarity in pattern indicates that VYSs of all 3 days have similar capacity for aerobic metabolism based on the pyridine nucleotide response. Freinkel et al. ('84) showed that the rat embryo undergoes a transition from anaerobic to aerobic energy metabolism in the developmental period from day 10 to day 11. It is, however, not specified if the VYS and the embryo proper undergo parallel changes with respect to aerobic metabolic capacity during this time or if aerobic capacity matures earlier in the VYS. Since the rat VYS is more directly exposed to the relatively oxygen rich maternal circulation and secretions, it would seem reasonable for the VYS to develop aerobic metabolic capacity earlier than in tissues of the embryo proper which remain relatively hypoxic on GD 10. The change to aerobic metabolism in the embryo proper closely corresponds to the time period when an active vitelline and embryonic circulation becomes operational (Copp and Cockroft, '90), thus matching a potential increase in embryonic oxygen concentration with a metabolically more efficient Kreb's cycle.

Our observations appear to contradict the 
widely held notion that the GD 10 conceptus is still in an anaerobic state and, having immature mitochondria, would not be expected to behave as the GD 11 or 12 conceptuses by increasing surface fluorescence during hypoxia as we have shown (Fig. 3B). The determinations that the GD 10 conceptus is anaerobic are based on studies using either embryo or whole conceptuses (Freinkel et al., '84) and through analysis of mitochondrial maturation and function in the developing embryonic heart (Mackler et al., '71; Fantel et al., '90). These studies do not preclude, however, the possibility that differential spatial and temporal maturation of mitochondria is taking place between embryo and VYS, much like that shown for asymmetrical mitochondria maturation related to head defects produced from cocaine exposure in vitro (Fantel et al., '90). Through the novel application of the microfiberoptic methodology presented in this report we provide evidence that the VYS (from which surface measurements are made) may, indeed, develop aerobic metabolic capacity earlier in gestation than in tissues of the embryo proper. It is not yet known whether the embryo proper and VYS would react in the same way in response to hypoxia. The simultaneous use of two ultrasmall sensors, one on the surface of the VYS and the second on the surface of the embryo proper, could provide a means to evaluate these differences in the developing conceptus.

Phase I showed the greatest increase in the rate of fluorescence, possibly reflecting the reduction of total NAD $(\mathrm{H})$ stores, especially in the younger conceptuses. This rate of response decreased over time, the GD 12 rate being nearly one-half of the rates seen on GD 10. Phase II, on the other hand, showed little variation between the three ages. This secondary effect may indicate the activation of pathways resulting from interconversion, accumulation, or biosynthesis of reduced pyridine nucleotides or possibly the appearance of other fluorophores with similar excitation/emission spectra. It is important to note that this reproducible rise and then rapid return to baseline levels of the endogenous surface fluorescence signal (following return to normoxic conditions), indicates that very dynamic intracellular processes are operating in the VYS during the nitrogen exposure.

Surface fluorometry, as its name implies, provides most of its information from the surface layers of a particular tissue. Depending on the thickness and fluorescence quenching ability of the tissue, it may or may not be suitable for deep tissue measurements. As previously referenced, deep tissue fluorescence measurements of reduced pyridine nucleotides have been demonstrated in the human muscle during exercise (Guezennec et al., '91). The fluorescence response that is monitored only reflects tissue conditions or damage which result from the change in the reduced pyridine nucleotide pool. Presently it is not possible to distinguish the fluorescence response between $\mathrm{NADH}$ and NADPH, since both produce a fluorescence signal of identical amplitude with equimolar concentrations of the reduced species. Furthermore, the $\operatorname{NAD}(\mathrm{P})^{+}$ $\mathrm{NAD}(\mathrm{P}) \mathrm{H}$ ratio cannot be obtained from this technique alone. With the combined use of analytical pyridine nucleotide quantitation techniques, however, it should be possible to correlate the fluorescence response to the actual changes in oxidized and reduced species in both VYS and embryo. Alterations in redox ratios could also be calculated under the various experimental conditions of chemical exposure or environmental extreme.

There are many advantages to using this MFOP system. First, the rat conceptus is kept intact, viable, and can be reintroduced to whole embryo culture following non-invasive fiberoptic monitoring, in order to evaluate additional parameters, such as morphologic and metabolic endpoints. The data is acquired in real time and on a continuous basis, thereby avoiding the necessity of obtaining static measurements requiring sacrifice of the animal and possible introduction of artifacts due to preparation. The rat conceptus also serves as its own experimental control, thus reducing the number of animals required to complete a study. Additional benefits of the MFOP system include its versatility and the ability to use exogenous customized fluorescence probe substrates to monitor other biochemical reactions or physiological processes. By changing the appropriate fiberoptic cutoff filters, matched to a particular fluorophore's excitation/emission spectrum, it should provide additional information without system modifications. The use of this fiberoptic technology has many potential applications, from drug and toxicology testing, 
evaluation of physiological and developmental function, and investigations into mechanisms of teratology.

\section{ACKNOWLEDGMENTS}

This work was supported by grant $15-179$ from the March of Dimes Birth Defects Foundation, training grant ES07062-12 and grant ES05235 from NIH, and a grant from the Rackham School of Graduate Studies, University of Michigan.

\section{LITERATURE CITED}

Belinsky, S.A., Reinke, L.A., Scholz, R., Kauffman, F.C., and Thurman, R.G. (1985) Rates of pentose cycle flux in perfused rat liver. Evaluation of the role of reducing equivalents from the pentose cycle for mixed-function oxidation. Mol. Pharmacol., 28:371376.

Berry, M.N., Hamilton, R.L., Severinghaus, E.M., and Williams, M.C. (1974) A simple and inexpensive membrane "lung" for rat liver perfusion. In: Regulation of Hepatic Metabolism. R. Lundquist and N. Tygstrup, eds. Alfred Benzon Symposium VI. Munksgaard, Copenhagen, Denmark, pp. 790-795.

Chance, B., Cohen, P., Joebis, F., and Schoener, B, (1962) Intracellular oxidation-reduction states in vivo. Science, 137:499-508.

Copp, A.J., Cockroft, D.L. (1990) Postimplantation mammalian embryos: A practical approach. IRL, Oxford University Press, New York.

Earle, W., Schilling, E., Stark, T., Strauss, N., Brown, M., and Shelton, E. (1943) Production of malignancy in vitro: The mouse fibroblast cultures and changes seen in the living cells. J. Natl. Cancer Inst., 4:167.

Fantel, A.G., Juchau, M.R., Burroughs-Gleim, C.J., and Person, R.E. (1989) Studies on mechanisms of niridazole-elicited embryotoxicity. Evidence that oxygen depletion plays a role in dysmorphogenicity. Teratology, 39:234-251.

Fantel, A.G., Person, R.E., Burroughs-Gleim, C.J., and Mackler, B. (1990) Direct embryotoxicity of cocaine in rats: Effects on mitochondrial activity, cardiac function, and growth and development in vitro. Teratology, 42:35-43.

Fantel, A.G., Person, R.E., Burroughs-Gleim, C.J., Shephard, T.H., Juchau, M.R., and Mackler, B. (1991) Assymetric development of mitochondrial activity in rat embryos as a determinant of the defect patterns induced by exposure to hypoxia, and redox cyclers in vitro. Teratology, 44:355-362.

Foster, E., DeJong, D., Connely, C., Apstein, C.S. (1984) Failure of nifedipine and reperfusion to reduce infarct size relative to region at risk as measured by NADH fluorophotography. Circulation, 70(3):506-512.

Freeman, S.J., Coakley, M., and Brown, N.A. (1987) Post-implantation embryo culture for studies of ter- atogenesis. In: Biochemical Toxicology: A Practical Approach K. Snell and B. Mullock, eds. IRL Press. New York, pp. 83-107.

Freinkel, N., Lewis, N.J., Akawawa, S., Roth, S.I., and Gorman, L. (1984) The honeybee syndrome-Implications of the teratogenicity of mannose in rat-embryo culture. N. Engl. J. Med., 310:223-230.

Gillet, P. (1981) Calculus and analytical geometry. D.C. Heath and Company, Lexington, Massachusetts.

Guezennec, C.Y., Lienhard, F., Lousy, F., Renault, G., Tusseau, M.H., Portero, P. (1991) In situ NADH laser fluorimetry during muscle contraction in humans. Eur. J. Appl. Physiol., 63(1):36-42.

Gupta, M., Gulamhusein, A.P., and Beck, F. (1982) Morphometric analysis of the visceral yolk sac endoderm in the rat in vivo and in vitro. J. Reprod. Fertil., 65: 239-245.

Harris, C., and Thurman, R.G. (1986) A new method to study glutathione adduct formation in periportal and pericentral regions of the liver lobule by micro-reflectance spectrophotometry. Mol. Pharmacol., 29:88-96.

Hiranruengchok, R., and Harris, C. (1993) Glutathione oxidation and embryotoxicity elicited by diamide in the developing rat conceptus in vitro. Toxicol. Appl. Pharmacol., 120(1):62-71.

Ji, S., Chance, B., Nisnili, K., Smith, T., and Rich, T. (1979) Micro-light guides, a new method for measuring tissue fluorescence and reflectance. Am. J. Physiol: 236:C144-C156.

Ji, S., Lemasters, J.L., and Thurman, R.G. (1980) A non-invasive method to study metabolic events within sublobular regions of hemoglobin-free perfused liver. FEBS Lett., 113:37-41.

Kapany, N.S. (1967) Fiber Optics: Principles and Applications. New York, Academic Press.

Mackler, B., Grace, R., and Duncan H.M. (1971) Studies of mitochondrial development during embryogenesis in the rat. Arch. Biochem. Biophys., 144:603-610.

Minezaki, K.K., Shinozaki, Y., Nakazawa, H., Yamada, Y., Okino, H. (1990) The detection of technical failures in perfused heart with ischemia and reperfusion by epicardial NADH fluorescence. Heart Vessels, 514 ! 193-197.

Olson, M.J., and Thurman, R.G. (1984) Endogenous ketogenesis in periportal and pericentral regions of the liver lobule. Fed. Proc. U.S.A., 43:1866.

Pappajohn, D., Penneys, R., and Chance, B. (1972 NADH spectrofluorometry of rat skin. J. Appl. Physiol., 33(5):684-687.

Sies, H. (1982) Nicotinamide nucleotide compartmentation. In: Metabolic Compartmentation. H. Sies, ed. Academic Press, London, pp. 205-231.

Thurman, R.G., and Lemasters, J.I. (1988) New microoptical methods to study metabolism in periportal and pericentral regions of the liver lobule. Drug Metab. Rev., 19(3\&4):263-281.

Todd, J.C., Sanford, A.H., Davidson, I., eds. : 1979) Chinical Diagnosis and Management by Laboratory Methods: Selection of Culture Systems. Vol II:1821. W.B. Saunders Co., Philadelphia, PA. 